$51.1 \%)$ or not $(n=46,48.9 \%)$ according to Samson and Scott criteria. Chi-squared test, t-test, and univariate/multivariate Cox regression were used. Survival curves were plotted via the Kaplan-Meier method, whilst survival differences were examined via the log-rank test for categorical variables or Cox regression for continuous variables. All reported p-values were two-tailed. Statistical significance was set at p-value $<0.05$. The statistical analysis was performed using Stata version 16.1 (Stata Corporation, TX, USA).

Results Women with OCCC arising from endometriosis had significantly lower levels of pre-operative CA-125 (434.63 \pm 1135.57 Vs $867.30 \pm 1609.67$, p-value $=0.02$ ) and significantly lower incidence of post-operative residual disease (RD) (p-value=0.02). Age, post-menopausal status, FIGO stage and incidence of capsule rupture were not statistically significant. The mean overall survival (OS) and overall progression free survival (PFS) were 86.35 (95\% CI 69.47 103.22) and 115.97 (95\% CI 98.77 - 133.17) months, respectively. The presence of endometriosis did not affect neither the OS $(87.99 \mathrm{Vs} 75.30$, p-value $=0.25)$ nor the PFS (111.13 Vs 117.42, p-value=0.48). In univariate analysis, the FIGO stage II-IV and RD were correlated with poorer OS, whilst capsule rupture (CR) with poorer PFS. In multivariate analysis, FIGO stage $[\mathrm{HR}=2.86 \quad(95 \%$ CI 1.47 5.55), p-value $=0.002]$ and $\mathrm{RD}[\mathrm{HR}=2.52$ (95\% CI 1.28 4.94, $\mathrm{p}$-value $=0.007$ ) were found independent predictors for OS, whilst CR $[\mathrm{HR}=0.3 \quad(95 \%$ CI 0.11 - 0.82), pvalue $=0.02]$ for PFS, respectively. No factors affected OS after stratification by stage.

Conclusion In this cohort concurrent endometriosis was not a predictive factor for the survival of OCCC women. Further studies are warranted to ascertain whether OCCC with or without coexisting endometriosis develop via distinct pathogenic pathways.

Disclosures Nil to disclose.

\section{EFFICACY AND SAFETY OF NIRAPARIB IN OLDER PATIENTS (PTS) WITH ADVANCED OVARIAN CANCER (OC): RESULTS FROM THE PRIMA/ENGOT-OV26/GOG- 3012 TRIAL}

${ }^{1}$ Hanna Dahlstrand, ${ }^{2}$ Bhavana Pothuri, ${ }^{3}$ Whitney Graybill, ${ }^{4}$ Thibault de La Motte Rouge, ${ }^{5}$ Colleen Mccormick, ${ }^{6}$ Florian Heitz, ${ }^{7}$ Jean-Francois Baurain, ${ }^{8}$ LI Yong, ${ }^{9}$ Bradley J Monk,

${ }^{10}$ Antonio González-Martin. 'Uppsala University; Department of Immunology, Genetics and Pathology; ${ }^{2}$ Gynecologic Oncology Group (Gog); Perlmutter Cancer Center, Nyu Langone Health; Department of Obstetrics/Gynecology; ${ }^{3}$ Medical University of South Carolina; ${ }^{4}$ Eugene Marquis Cancer Center, ${ }^{5}$ Legacy Medical Group Gynecologic Oncology; ${ }^{6}$ Ago Study Group; Charité - Universitätsmedizin Berlin, Humboldt-Universität zu Berlin, Berlin Institute of Health; Department of Gynecology; ' Université Catholique de Louvain and Cliniques Universitaires Saint-Luc; ${ }^{8}$ Glaxosmithkline; ${ }^{9}$ Arizona Oncology (US Oncology Network), University of Arizona College of Medicine; ${ }^{10}$ Grupo Español de Investigación En Cáncer de Ovario (Geico); Clínica Universidad de Navarra; Medical Oncology Department

\subsection{6/ijgc-2020-ESG0.123}

Introduction/Background The PRIMA/ENGOT-OV26/GOG3012 (PRIMA) trial showed that niraparib significantly improves progression-free survival (PFS) in pts with newly diagnosed advanced OC that responded to first-line platinumbased chemotherapy (CT) (hazard ratio [HR] 0.62; 95\% CI 0.50-0.76). Here we discuss the impact of age on efficacy and safety of niraparib.

Methodology This double-blind, placebo (PBO)-controlled phase 3 trial evaluated niraparib in pts with newly diagnosed, advanced, high-grade serous or endometrioid ovarian, primary peritoneal, or fallopian tube cancer with a complete or partial response to first-line platinum-based CT. Pts were randomised 2:1 to receive either a fixed starting dose (FSD) of $300 \mathrm{mg}$ niraparib or PBO QD. A protocol amendment introduced an individualised starting dose (ISD): $200 \mathrm{mg}$ QD in pts with bodyweight $<77 \mathrm{~kg}$ or platelet count $<150,000 /$ $\mu \mathrm{L}$ or $300 \mathrm{mg}$ QD for all others. Pts were dichotomized by age group $<65$ vs $\geq 65$ years old (yo) to analyse efficacy and

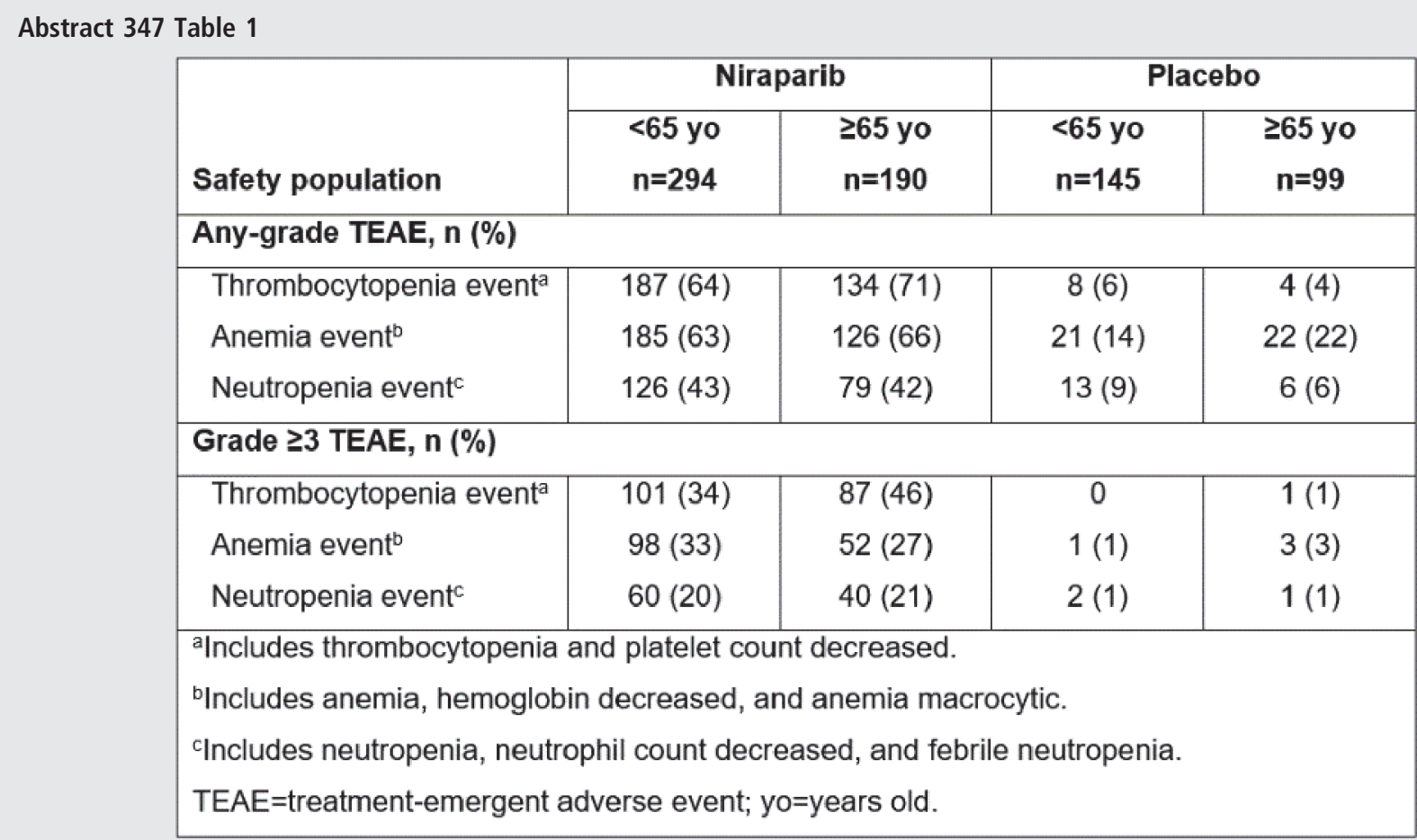


safety of niraparib vs $\mathrm{PBO}$ in older patients. The primary endpoint was PFS assessed by blinded independent central review.

Results Of 733 enrolled pts, 444 were $<65$ yo (297 niraparib, $147 \mathrm{PBO}$ ), and 289 were $\geq 65$ yo (190 niraparib, $99 \mathrm{PBO}$ ). Efficacy was comparable in pts $<65$ yo (HR 0.61; 95\% CI $0.47-0.81$ ) and $\geq 65$ yo (HR $0.53 ; 95 \%$ CI $0.39-0.74$ ) who received niraparib compared with $\mathrm{PBO}$. Any-grade and grade $\geq 3$ treatment emergent adverse events were similar across age groups (table 1). Grade $\geq 3$ thrombocytopenia events in pts $<65$ yo were reported in $43 \%$ of pts receiving a FSD and $18 \%$ of pts receiving ISD. In pts $\geq 65$ yo, the values were $57 \%$ and 26\%, respectively. Patient reported outcomes (PROs) and quality of life (QOL) were similar in both age groups as assessed by FOSI and EQ-5D-5L.

Conclusion Niraparib efficacy, safety, and QOL were similar in compared age groups. Implementation of an ISD regimen improved rates of grade $\geq 3$ thrombocytopenia events in older pts.

\section{Disclosures Funding: GlaxoSmithKline}

NCT number: NCT02655016

Encore statement: This data is presented on behalf of the original authors with their permission. Presented at the European Society for Medical Oncology (ESMO) Annual Meeting, September 19-21, 2020, Virtual.

Dr. Dahlstrand reports personal fees from AstraZeneca and Roche.

Dr. Pothuri reports grants, personal fees and non-financial support from GSK; Advisory Board fees from AstraZeneca and Clovis Oncology.

Dr. Graybill reports personal fees from GSK.

Dr. McCormick has nothing to disclose.

Dr. de La Motte Rouge reports personal fees and nonfinancial support from ASTRAZENECA, MSD, and Roche; personal fees from Clovis Oncology and GlaxoSmithKline; and grants, personal fees, and non-financial support from Pfizer.

Dr. Heitz reports non-financial support from NewOncology; Personal fees from Roche, AstraZeneca, Clovis, Tesaro, and PharmaMar.

Dr. Monk reports consulting and advisory role at Merck, GSK, Roche/Genentech, AstraZeneca, Advaxiz, Cerulean Pharma, Amgen, Immunogen, NuCana BioMed, Clovis Oncology, Pfizer, Mateon Therapeutics, Precision Oncology, Perthera, Abbvie, Myriad Pharmaceuticals, Incyte, VBL Therapeutics, Takeda, Samumed, Oncomed, OncoSec, ChemoID, Geistlich Pharma, Eisai and Chemocare; Speakers' bureau at Roche/Genentech, AstraZeneca, Janssen, Clovis Oncology and GSK; Honoraria from Merck, GSK, Roche/Genentech, AstraZeneca, Advaxis, Immunogen, NuCana BioMed, Clovis Oncology, Pfizer, Mateon Therapeutics, Precision Oncology, Pethera, Abbvie, Myriad Pharmaceuticals, Incyte, Janssen, Amgen, Genmab, Samumed, Takeda, VBL Therapeutics, Puma Biotechnology, Immunomedics, Conjupro Biotherapeutics, Agenus, OncoQuest, ChemoID, Geistlich Pharma, Eisai and Chemocare; and Research funding from Novartis, Amgen, Genentech, Lilly, Janssen, Array BioPharma, GSK, Morphotek, Pfizer, Advaxis, AstraZeneca, Immunogen, Regeneron, and Nucana.

Dr. González-Martín reports personal fees and non-financial support from AstraZeneca; Grant and personal fees from GSK, Clovis Oncology, Roche Holding AG, Merck \& Co., Inc., Genmab, INMUNOGEN, Pharma Mar, S.A., and Oncoinvent AS.

Drs Li and Gupta are employees of GlaxoSmithKline.

\section{4} EFFICACY OF NIRAPARIB THERAPY IN PATIENTS WITH NEWLY DIAGNOSED ADVANCED OVARIAN CANCER BY BRCAWT STATUS: PRIMA/ENGOT-OV26/GOG-3012 STUDY

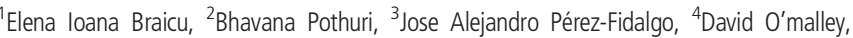
${ }^{5}$ Brigitte Honhon, ${ }^{6}$ Whitney Graybill, ${ }^{7}$ Michel Fabbro, ${ }^{8}$ Hanna Dahlstrand, ${ }^{9}$ Divya Gupta, ${ }^{10}$ Bradley J Monk. ${ }^{1}$ Charite Medical University; ${ }^{2}$ Gynecologic Oncology Group (Gog); Perlmutter Cancer Center, Nyu Langone Health; Department of Obstetrics/Gynecology: ${ }^{3}$ Incliva University Hospital of Valencia, Ciberonc; Department of Medical Oncology; ${ }^{4}$ The Ohio State University - James CCC; ${ }^{5}$ Grand Hôpital De Charleroi; Department of Oncology; ${ }^{6}$ Gog; Medical University of South Carolina; Gynecologic Oncology; ${ }^{7}$ Institut du Cancer de Montpellier; ${ }^{8}$ Uppsala University; Uppsala University Hospital; Department of Immunology, Genetics, and Pathology; Department of Oncology; ${ }^{9}$ Glaxosmithkline; ${ }^{10}$ Arizona Oncology (US Oncology Network), University of Arizona College of Medicine

\subsection{6/ijgc-2020-ESGO.124}

Introduction/Background Niraparib is a poly(ADP-ribose) polymerase inhibitor approved for maintenance treatment of patients with newly diagnosed advanced or platinum-sensitive, recurrent ovarian cancer (OC). Niraparib is also approved in the United States for the treatment of patients with OC who received $\geq 3$ lines of therapy and whose cancer is either BRCA mutated or homologous recombination deficient (HRd) platinum-sensitive disease. The PRIMA/ENGOT-OV26/GOG3012 trial showed that niraparib significantly improves progression-free survival (PFS) in patients with newly diagnosed advanced OC that responded to first-line platinum-based chemotherapy (hazard ratio, $0.62 ; 95 \%$ CI, 0.50-0.76). Here we report the efficacy of niraparib in patients by BRCA wild-type (BRCAwt) status.

Methodology This double-blind, placebo-controlled, phase 3 trial evaluated niraparib in patients with newly diagnosed, advanced, high-grade serous or endometrioid ovarian, primary peritoneal or fallopian tube cancer with a complete response (CR) or partial response (PR) to first-line platinumbased chemotherapy. Patients were stratified by best response to the first-line chemotherapy (CR/PR), receipt of neoadjuvant chemotherapy (yes/no), and homologous recombination status (deficient/proficient and not determined). Patients were randomised 2:1 to receive either niraparib or placebo once daily. The primary endpoint of PFS, assessed by blinded independent central review, was analysed using a stratified log-rank test and Cox proportional hazards model and hierarchically tested in HRd patients, then the overall population. BRCA and HRd status were determined by tumour samples at screening via the myChoice ${ }^{\circledR}$ test (Myriad, Salt Lake City, Utah). The prespecified BRCAwt subgroup PFS analysis was performed using a stratified log-rank test and Cox proportional hazards model and using Kaplan-Meier methodology. BRCAwt subgroups included the intention-totreat/BRCAwt (all patients who were homologous recombination not determined [HRnd]/BRCAwt, HRd/BRCAwt, and homologous recombination proficient [HRp]/BRCAwt); subgroup analyses on the HRd/BRCAwt and HRp/BRCAwt were performed.

Results Of 733 randomised patients, 473 (64.5\%) had BRCAwt tumours (74 patients had unknown BRCA status). Of these 473, 150 (31.7\%) had HRd/BRCAwt tumours, $249(52.6 \%)$ had HRp/BRCAwt tumours, and 74 (15.7\%) had HRnd/BRCAwt tumours. Niraparib-treated patients with BRCAwt tumours had a clinically meaningful PFS benefit regardless of homologous recombination status (table 1). 